COMMUNICATIONS IN

ANALYSIS AND GEOMETRY

Volume 11, Number 3, 425-439, 2003

\title{
Minimal Spheres of Arbitrarily High Morse Index
}

\author{
Joel Hass, Paul Norbury, and J. Hyam Rubinstein \\ We construct a smooth Riemannian metric on any 3-manifold with \\ the property that there are genus zero embedded minimal surfaces \\ of arbitrarily high Morse index.
}

\section{Introduction.}

In connection with the spherical space form problem, Pitts and Rubinstein [7] raised the question of whether embedded minimal surfaces of fixed genus in a three-manifold have bounded Morse index. An area bound on minimal surfaces of fixed genus would imply an index bound, however in [3] it was shown that on any three-manifold there exists an open set of metrics for which there are embedded minimal tori of arbitrarily large area. These minimal tori have Morse index zero. In this paper we construct metrics for which there are embedded minimal spheres of arbitrarily large Morse index.

We use the techniques from [5] to study circle invariant metrics and reduce the problem to one of finding a family of geodesics of arbitrarily large index on a surface equipped with a metric that degenerates at the boundary.

Theorem 1. On any three-manifold there exists a metric for which there are embedded minimal spheres of arbitrarily large Morse index. On the three-sphere it can be chosen to have non-negative Ricci curvature.

The Ricci curvature of each example of the theorem is non-positive somewhere. Each of the metrics on the three-sphere constructed by the theorem possesses an arbitrarily small perturbation to a positive Ricci curvature metric. If a manifold has a metric of positive Ricci curvature then there is an area bound on the embedded minimal spheres $[1,4]$ and hence a bound on their index. Thus the property of the existence of embedded minimal spheres of arbitrarily large Morse index is destroyed by the perturbations.

Using completely different methods, Colding and Hingston [2] have constructed metrics on any three-manifold with embedded minimal tori of arbitrarily high index. This is complementary to the work here since the

\footnotetext{
${ }^{1} \mathrm{PN}$ and JHR would like to acknowledge the support of the Australian Research Council.
} 
conjecture of Pitts and Rubinstein [7] requires a Morse index bound on a compactification of the space of embedded tori which includes both spheres and tori.

\section{The quotient construction.}

Consider the product metric on $\mathbb{R} \times S^{2}$ given by the Euclidean line times the round two-sphere:

$$
d s_{0}^{2}=d r^{2}+d \varphi^{2}+\sin ^{2} \varphi d \theta^{2}
$$

where $\varphi \in[0, \pi]$ and $\theta \in[0,2 \pi]$ are spherical coordinates on the two-sphere. This is invariant under rotations by $\theta$, and the quotient metric is

$$
d s_{1}^{2}=d r^{2}+d \varphi^{2}
$$

defined on $\mathbb{R} \times[0, \pi]$. Geodesics on the quotient surface have little to do with minimal surfaces upstairs since the lengths of the orbit circles vary. Instead, following [5] we adjust the metric by $\sin \varphi$, the lengths of the orbit circles:

$$
d s^{2}=\sin ^{2} \varphi\left(d r^{2}+d \varphi^{2}\right)
$$

This is a metric on $\mathbb{R} \times[0, \pi]$ degenerate on the boundary, and its geodesics pull back to minimal surfaces in $\mathbb{R} \times S^{2}$. Its curvature is $1 / \sin ^{4} \varphi$.

The equation for geodesics is given by:

$$
\begin{gathered}
\ddot{r}=-2 \frac{\cos \varphi}{\sin \varphi} \dot{r} \dot{\varphi} \\
\ddot{\varphi}=\frac{\cos \varphi}{\sin \varphi}\left(\dot{r}^{2}-\dot{\varphi}^{2}\right)
\end{gathered}
$$

and (2) is equivalent to $d(\ln \dot{r}) / d t=-2 d(\ln \sin \varphi) / d t$ so

$$
\dot{r}=\frac{c}{\sin ^{2} \varphi}
$$

for a constant $c$. The constant $c$ is equal to zero precisely when the geodesic is vertical ( $r=$ constant) and thus meets the boundary. For all other geodesics $\dot{r}$ is always positive or always negative. The speed $\left(\dot{r}^{2}+\dot{\varphi}^{2}\right) \sin ^{2} \varphi$ is a conserved quantity and we may set it to be 1 . Thus, $|\dot{r}| \leq 1 / \sin \varphi$ so from (4),

$$
0 \leq|c| \leq \sin \varphi
$$




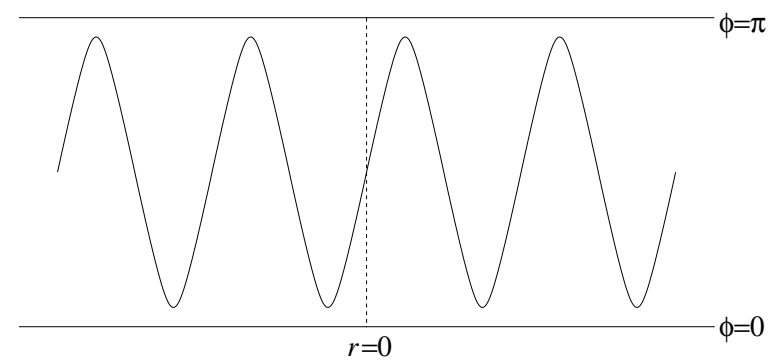

Figure 1: The degenerate boundary repels geodesics.

For non-vertical geodesics $|c|$ is strictly positive so $\varphi$ is bounded away from 0 and $\pi$, or in other words the boundary repels the geodesic - see Figure 1. The maximum and minimum values of $\varphi$ of the geodesic satisfy $\sin \varphi=|c|$ and the geodesic is periodic by the translation and reflection invariance of $d s^{2}$.

This is much like the construction in [5] for the round metric on $S^{3}$. The major difference is that the period of the geodesics can be made arbitrarily small in our case whereas there is a lower bound for the period in [5].

Lemma 2.1. The period, in $r$, of the geodesic with minimum $\sin \varphi=c>0$ is less than $2 \pi \sqrt{2 c /(1+c)}$.

Proof. From (4) and $\left(\dot{r}^{2}+\dot{\varphi}^{2}\right) \sin ^{2} \varphi=1$ we get

$$
\left(\frac{d r}{d \varphi}\right)^{2}=\frac{\dot{r}^{2}}{\dot{\varphi}^{2}}=\frac{c^{2}}{\sin ^{2} \varphi-c^{2}} .
$$

By symmetry one quarter of a period of the geodesic is given by the following integral:

$$
\begin{aligned}
r & =\int_{\sin \varphi=c}^{\pi / 2} \frac{c}{\sqrt{\sin ^{2} \varphi-c^{2}}} d \varphi \\
& =c \int_{c}^{1} \frac{d u}{\sqrt{\left(u^{2}-c^{2}\right)\left(1-u^{2}\right)}} \\
& <\sqrt{\frac{c}{2(1+c)}} \int_{c}^{1} \frac{d u}{\sqrt{(u-c)(1-u)}}=\pi \sqrt{\frac{c}{2(1+c)}}
\end{aligned}
$$


The energy functional on the space of paths between points $p$ and $q$ in a manifold $M$ is defined to be the integral of the square of the norm of the derivative along the path. If we simply integrate the norm along the path then this gives the length functional. The critical points of the energy functional coincide with the critical points of the length functional, and thus are the geodesics. The Hessian of the energy functional is a bilinear form with finite nullity and index - the respective dimensions of the null space and the largest subspace on which the Hessian is negative definite. It is non-degenerate if the nullity is zero.

For generic $p$ and $q$ the energy functional is Morse. That is, its critical points are non-degenerate [6]. Along any geodesic $\gamma(t)$, the energy functional can be defined on an initial interval $t \in[0, \tau]$ say. The geodesic is nondegenerate for generic $\tau$, and its index remains the same for nearby generic values of $\tau$. It is important to note that the energy functional is defined on a different set of paths for each $\tau$ and in particular that the tangent spaces change - the infinitesimal variations are given by vector fields that vanish at 0 and $\tau$. On each side of a non-generic value of $\tau$ the index of the geodesics can be related, leading to the Morse index theorem.

The points $p$ and $q$ are conjugate along a geodesic $\gamma$ joining them if the nullity of $\gamma$ is non-zero, or in other words the geodesic is a degenerate critical point of the energy functional. Thus along $\gamma$ there exists a null vector field or equivalently a Jacobi vector field that vanishes at $p$ and $q$. A Jacobi vector field is a vector field $W$ defined along $\gamma$ that satisfies the Jacobi equation:

$$
D^{2} W / d t^{2}+R(W, V) V=0
$$

where $D$ is the covariant derivative along $\gamma, V=d \gamma / d t$ and $R(u, v)=$ $D_{v} D_{u}-D_{u} D_{v}$ is the curvature of the metric. More generally, any two points on $\gamma$ are conjugate along $\gamma$ if there exists a Jacobi vector field along $\gamma$ that vanishes at the two points.

The Morse index theorem states that the index of a geodesic is equal to the number of points conjugate to one of its endpoints. The conjugate points are counted with multiplicity which is 1 on a surface.

On the surface with metric (1) there are geodesics with arbitrarily high index. This uses Lemma 2.1 which tells us that for any $N \in \mathbb{Z}$, when $c$ is small enough the geodesic with minimum $\sin \varphi=c$ meets $\varphi=\pi / 2$ at least $N$ times, and the next lemma in which we show that this gives a lower bound of $N$ for the Morse index of the geodesic.

Lemma 2.2. The index of a geodesic is bounded below by half the number of times it meets $\varphi=\pi / 2$. 
Proof. Along any geodesic $\gamma$ with minimum $\sin \varphi=c$, choose a piece $\gamma^{0}$ that travels a full period. In the next paragraph we show there exists a pair of conjugate points along $\gamma^{0}$, and thus by the Morse index theorem, the index of $\gamma^{0}$ is at least 1 . That is, there exists a vector field along $\gamma^{0}$ that vanishes at its endpoints and on which the Hessian of the energy functional is negative definite. Extend this to $\gamma$ by setting it to be zero outside $\gamma^{0}$. Do this along consecutive pieces of the geodesic $\gamma$ to get a set of vector fields on which the Hessian of the energy functional is diagonal and negative definite. Hence the index of a geodesic is bounded below by half the number of times it meets $\varphi=\pi / 2$.

Since the Gaussian curvature of the metric is bounded below by 1 we can use the Rauch comparison theorem to deduce that the distance between conjugate points is at most $\pi$. The length of $\gamma^{0}$ is given by:

$$
\begin{aligned}
l & =2 \int_{\varphi=\pi / 2}^{\sin \varphi=c} \sin \varphi \sqrt{1+(d r / d \varphi)^{2}} d \varphi \\
& =2 \int_{\varphi=\pi / 2}^{\sin \varphi=c} \frac{\sin ^{2} \varphi}{\sin ^{2} \varphi-c^{2}} d \varphi \\
& =2 \int_{0}^{\pi / 2} \sqrt{\cos ^{2} \alpha+c^{2} \sin ^{2} \alpha} d \alpha \\
& \geq 2 \int_{0}^{\pi / 2} \cos \alpha d \alpha=2
\end{aligned}
$$

where we have used the substitution $\sin \varphi=\sin \alpha \sqrt{1-c^{2}}$. Thus the length of a geodesic along its full period is greater than 4 so contains conjugate points by the Rauch comparison theorem.

We can improve the previous lemma and bound the index of a geodesic below by the number of times it meets $\varphi=\pi / 2$. A minimax argument shows that the index of a piece of the geodesic that travels from $\varphi=\pi / 2$ to its minimum and back - half the period-is 1 . This uses a sequence of sweepouts between two index zero geodesics - the horizontal geodesic along $\varphi=\pi / 2$ and the degenerate piecewise geodesic consisting of two vertical geodesics from $\varphi=\pi / 2$ to $\varphi=0$ together with a path (of zero length) along the boundary. The minimax is an index 1 geodesic. 


\section{Perturbed metric.}

Perturb the product metric on $\mathbb{R} \times S^{2}$ to get

$$
d s_{0}^{2}=d r^{2}+\lambda(r)\left(d \varphi^{2}+\sin ^{2} \varphi d \theta^{2}\right)
$$

where $\lambda(r)=1$ for $r \leq 0$ and $\lambda^{\prime}(r)<0$ for $r>0$. The metric is defined only for $r$ satisfying $\lambda(r) \geq 0$. We also require that $\lambda^{\prime}(r)=0$ when $\lambda(r)=0$ so that the metric is smooth as $\lambda(r) \rightarrow 0$.

The metric on $\mathbb{R} \times[0, \pi]$ degenerate on the boundary is

$$
d s^{2}=\lambda(r) \sin ^{2} \varphi\left(d r^{2}+\lambda(r) d \varphi^{2}\right) .
$$

The equation for geodesics is given by:

$$
\begin{aligned}
& \ddot{r}=-2 \frac{\cos \varphi}{\sin \varphi} \dot{r} \dot{\varphi}-\frac{\lambda^{\prime}(r)}{2 \lambda(r)} \dot{r}^{2}+\lambda^{\prime}(r) \dot{\varphi}^{2} \\
& \ddot{\varphi}=\frac{\cos \varphi}{\sin \varphi}\left(\frac{1}{\lambda(r)} \dot{r}^{2}-\dot{\varphi}^{2}\right)-2 \frac{\lambda^{\prime}(r)}{\lambda(r)} \dot{r} \dot{\varphi}
\end{aligned}
$$

or, alternatively

$$
\frac{d^{2} r}{d \varphi^{2}}=-\frac{1}{\lambda(r)} \frac{\cos \varphi}{\sin \varphi}\left(\frac{d r}{d \varphi}\right)^{3}+\frac{3}{2} \frac{\lambda^{\prime}(r)}{\lambda(r)}\left(\frac{d r}{d \varphi}\right)^{2}-\frac{\cos \varphi}{\sin \varphi} \frac{d r}{d \varphi}+\lambda^{\prime}(r)
$$

Put

$$
\lambda(r)=\cos ^{2} r
$$

for $0 \leq r \leq \pi / 2$, and $\lambda \equiv 1$ for $r<0$. This gives a $C^{1}$ metric. A family of solutions to $(9)$ is

$$
\cos \varphi=\frac{\tan r}{\tan \kappa}
$$

for $r \geq 0$ and a constant $\kappa \in[0, \pi]$. We uniquely continue to a periodic solution on $r<0$. Each of these geodesics meets the boundary at right angles, at $(\kappa, 0)$ or $(\pi-\kappa, \pi)$, and passes through the focal point $(r, \varphi)=$ $(0, \pi / 2)$ at an angle of $\theta(\kappa)$ with the line $r=0$. As $\kappa \rightarrow 0, \theta(\kappa) \rightarrow 0$.

For a given $\epsilon>0$, countably many of these solutions meet $(r, \varphi)=$ $(-\epsilon, \pi / 2)$ since as $\theta(\kappa)$ gets smaller, so does the period and geodesics with a period of $2 /(n \epsilon)$ for $n \in \mathbb{Z}$ meet $r=-\epsilon$ and $r=0$ at $\varphi=\pi / 2$. If we reflect the metric in $r=-\epsilon / 2$,

$$
\lambda(r)=\left\{\begin{array}{cc}
\cos ^{2}(-r-\epsilon) & r \leq-\epsilon \\
1 & -\epsilon<r<0 \\
\cos ^{2}(r) & 0 \leq r
\end{array}\right.
$$


then we have a constructed an infinite family of geodesics with arbitrarily large index that run from the boundary to the boundary. These lift to compact minimal surfaces in $S^{3}$. The metric on $S^{3}$ is obtained from the round metric by cutting $S^{3}$ along a totally geodesic $S^{2}$ and gluing in the product $[-\epsilon, 0] \times S^{2}$ of the Euclidean metric times the round metric. The surfaces have arbitrarily large index since by [5] Jacobi fields lift to Jacobi fields upstairs and we can use a generalisation of the Morse index theorem by Simons [8] to get a lower bound for the index.

\section{Smooth perturbed metric.}

In this section we construct smooth metrics with similar properties to the $C^{1}$ example in the previous section. The main differences are that we do not have an explicit formula for the geodesics and there is no focal point. We call a geodesic that meets the boundary a boundary geodesic. For particular smooth functions $\lambda(r)$ defined on a neighbourhood of $r=0$ the following properties hold:

(i) Any boundary geodesic meets the boundary with known first and second derivatives.

(ii) If we squeeze at a faster rate (a condition on $\lambda^{\prime}(r)$ ) than for the $C^{1}$ example (10), then the geodesics (11) form barriers for the boundary geodesics.

(iii) There exist geodesics meeting all points of the boundary.

(iv) The boundary geodesics meet the line $r=0$ at arbitrarily small angles.

(v) There exists a countable set of boundary geodesics with arbitrarily high index that must meet the boundary again as in Figure 2.

(vi) The Ricci curvature of the metric on the three-sphere can be chosen to be non-negative.

In the remainder of this section we give the proofs of these properties. The precise meaning of barrier in (ii) is given in Lemma 4.2. Proposition 4.1 contains the proof of (iii). To prove (iv) we take geodesics meeting the boundary at arbitrarily small $r>0$ and use (ii) to show these geodesics meet the line $r=0$. A second derivative estimate shows that the geodesics remain close to vertical there. As the geodesics become more vertical, their period along $r<0$ becomes smaller and the index becomes larger. For (v) 


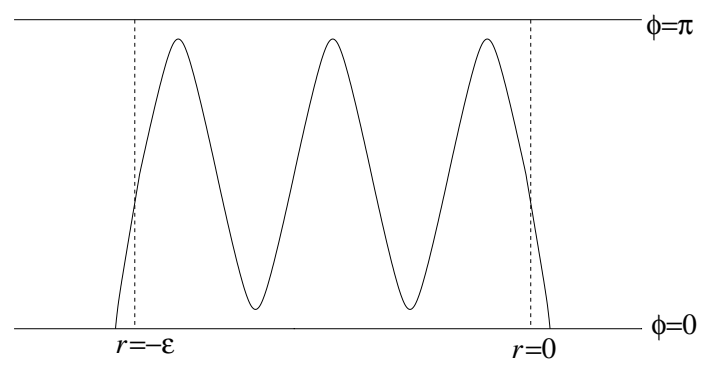

Figure 2: A geodesic meets the boundary twice.

we prove that a countable set meet the lines $r=-\epsilon$ and $r=0$ at the same value of $\varphi$ and same angle (with opposite sign.) These pull back to embedded minimal surfaces in $S^{3}$ of arbitrarily high index.

Lemma 4.1. There are geodesics meeting the boundary at $r>0$. They meet the boundary orthogonally, with second derivative $d^{2} r /\left.d \varphi^{2}\right|_{\varphi=0}=\lambda^{\prime}(r) / 2$ and each is the unique geodesic to meet its boundary point.

Proof. The shortest path from any point to the boundary is a geodesic. For the moment assume that $\lambda(r) \equiv 1$ for $r \leq 0$ (so Figure 2 is not correct for $r<-\epsilon$.) Such a path cannot cross $r=0$ since any geodesic that $\operatorname{crosses} r=0$ is not vertical so cannot meet the boundary on $r<0$. Thus it meets the boundary at $r>0$. For $\varphi$ small enough, there is a unique shortest path to the boundary by the uniqueness of a minimal disk upstairs with boundary given by a small circle. A geodesic meets the boundary orthogonally since upstairs the circle action splits the tangent space of the fixed point into the invariant tangent plane of the minimal surface and its orthogonal complement pointing in the direction of the fixed point set. In other words $d r / d \varphi=0$ at the boundary. The $\operatorname{limit}_{\lim } \lim _{\varphi} d^{2} r / d \varphi^{2}$ exists by smoothness of the minimal surface upstairs. It is equal to $\lim _{\varphi \rightarrow 0}(d r / d \varphi) / \sin \varphi$ and we can calculate it using (9):

$$
d^{2} r /\left.d \varphi^{2}\right|_{\varphi=0}=-d^{2} r /\left.d \varphi^{2}\right|_{\varphi=0}+\lambda^{\prime}(r)
$$

Any point on the boundary is met by at most one geodesic: if not then two such geodesics would lift to tangent minimal surfaces violating the maximum principle.

In the following lemma we prove that the geodesics from the $C^{1}$ example in the last section act as barriers for the geodesics of metrics satisfying (12). 
We refer to the former geodesics as leaves of a foliation, to reduce confusion when talking about two sets of geodesics. The argument is quite easy. We prove that a geodesic of a metric that squeezes fast enough can only be parallel from the left to a leaf of the foliation. A geodesic that moves away from the left of a leaf cannot return to that leaf since it would have to meet another leaf at a tangent from the right. Here left refers to the $(r, \varphi)$ cartesian plane with $r$ on the horizontal axis. The point $\left(r_{1}, \varphi\right)$ lies to the left of $\left(r_{2}, \varphi\right)$ when $r_{1}<r_{2}$.

Lemma 4.2. If

$$
\lambda^{\prime}(r)<-\sin 2 r
$$

for $r>0$ then a geodesic that meets the boundary at $(r, \varphi)=\left(r_{0}, 0\right)$ does not meet the curve $\cos \varphi=\tan r / \tan r_{0}$ in $r \in\left[0, r_{0}\right)$.

Proof. Along the leaf $\cos \varphi=\tan r / \tan r_{0}$, at $(r, \varphi)=\left(r_{0}, 0\right)$ we have $d r / d \varphi=0$ and $d^{2} r / d \varphi^{2}=-\cos r_{0} \sin r_{0}$. By Lemma 4.1 , a boundary geodesic meets $(r, \varphi)=\left(r_{0}, 0\right)$ with $d r / d \varphi=0$ and $d^{2} r / d \varphi^{2}<-\cos r_{0} \sin r_{0}$. Thus, near $\varphi=0$ the boundary geodesic lies to the left of the leaf $\cos \varphi=\tan r / \tan r_{0}$. The leaves $\cos \varphi=\tan r / \tan \kappa$ foliate the surface. If the boundary geodesic were ever to meet the leaf $\cos \varphi=\tan r / \tan r_{0}$ again then at some point it would be tangent to the foliation, from the right of a leaf.

If $\lambda^{\prime}(r)<-\sin 2 r$ then $\lambda(r)<\cos ^{2} r$ for $r>0$ since $\lambda(0)=1$. Along the boundary geodesic with direction $d r / d \varphi=-\sin \varphi \cos ^{2} r \tan \kappa$ (the tangent direction to the foliation), from (9) we have

$$
\begin{aligned}
\frac{d^{2} r}{d \varphi^{2}} & =\frac{\cos ^{4} r}{\lambda(r)}\left(\tan ^{2} \kappa-\tan ^{2} r\right)\left(\sin r \cos r+\frac{3}{2} \lambda^{\prime}(r)\right)+\sin r \cos r+\lambda^{\prime}(r) \\
& <\cos ^{2} r\left(\tan ^{2} \kappa-\tan ^{2} r\right)(-2 \sin r \cos r)-\sin r \cos r
\end{aligned}
$$

where we have replaced $\lambda^{\prime}(r)$ with $-\sin 2 r$ since its coefficient is positive, and $1 / \lambda(r)$ with $1 / \cos ^{2} r$ since its coefficient is negative. Compare this with the second derivative of the foliation $r_{\text {fol }}(\varphi)$

$$
\frac{d^{2} r_{\mathrm{fol}}}{d \varphi^{2}}=\sin r \cos r\left(1-2\left(1+\tan ^{2} \kappa\right) \cos ^{2} r\right)
$$

Then

$$
\frac{d^{2} r_{\mathrm{fol}}}{d \varphi^{2}}-\frac{d^{2} r}{d \varphi^{2}}>0
$$


Thus, if the geodesic is tangent to the foliation somewhere, the second derivative of the foliation is greater than that of the geodesic so the geodesic lies to the left of the leaf which contradicts the geometry described above, that it should lie to the right of the leaf.

An implicit form of the geodesic equations is given in (9). In the following lemma we use this to show that geodesics that meet the boundary at $\varphi=0$ near $r=0$ remain almost vertical.

Lemma 4.3. For small enough $r_{0}$, if a geodesic meets the boundary at $(r, \varphi)=\left(r_{0}, 0\right)$, then along the geodesic, whilst $r \geq 0, d r / d \varphi \leq 0$ and

$$
d^{2} r / d \varphi^{2}>\lambda^{\prime}(r)
$$

Proof. We begin by proving the lower bound on the second derivative when the first derivative is negative, and then use this to show that the first derivative remains negative along the boundary geodesic.

To get a lower bound on $d^{2} r / d \varphi^{2}$ we use the polynomial (9). By Lemma 4.2 , if a geodesic meets the boundary at $\varphi=0$, then along the geodesic $\cos \varphi / \sin \varphi>0$. This fact, together with $\lambda^{\prime}(r)<0$ shows that the equation (9) expresses $d^{2} r / d \varphi^{2}$ as a cubic polynomial in $d r / d \varphi$ with negative coefficients. We will arrange things so that it is a monotone cubic, then we have $d^{2} r / d \varphi^{2} \geq \lambda^{\prime}(r)$ for $d r / d \varphi<0$.

The polynomial is monotone when the discriminant of its derivative is negative. Thus, differentiate (9) with respect to $d r / d \varphi$. It is quadratic in $d r / d \varphi$ with discriminant

$$
\Delta=\frac{3}{\lambda(r)}\left(\frac{3 \lambda^{\prime}(r)^{2}}{4 \lambda(r)}-\frac{\cos ^{2} \varphi}{\sin ^{2} \varphi}\right) .
$$

This is negative for any $r$ when $\varphi$ is small enough since we require $\lambda^{\prime}(r)=0$ when $\lambda(r)=0$, so $\lambda^{\prime}(r)^{2} / \lambda(r)$ is bounded. (Its limit as $\lambda(r)$ vanishes is $\left.2 \lambda^{\prime \prime}(r).\right)$

We will show that when $r$ is small enough, (14) is negative for all $\varphi$ along the boundary geodesic. The lower side of the graph of $\Delta(r, \varphi)=0$ determines where the discriminant is negative, where the lower side means the side containing very small $\varphi$. It is sufficient to show that the graph of $\Delta(r, \varphi)=0$ lies entirely to one side of a leaf of the foliation $\cos (r)=\tan (r) / \tan \left(r_{0}\right)$ for $r_{0}$ small enough. Then we apply Lemma 4.2 to conclude that the boundary geodesics meeting $\varphi=0$ at $r \leq r_{0}$ lie below the leaf $\cos (r)=\tan (r) / \tan \left(r_{0}\right)$ and hence the discriminant is negative there. 
The graph of $\Delta(r, \varphi)=0$ meets $r=0$ at the focal point of the foliation, $(r, \varphi)=(0, \pi / 2)$. It meets it there horizontally, i.e. with $d r / d \varphi=\infty$, whereas, the leaf of the foliation $\cos (r)=\tan (r) / \tan \left(r_{0}\right)$ meets the foliation at an angle of $d r / d \varphi=-\tan \left(r_{0}\right)$. Thus, the graph of $\Delta(r, \varphi)=0$ initially lies above all of the leaves of the foliation. Furthermore, the graph of $\Delta(r, \varphi)=0$ only proceeds away from $r=0$, i.e. $d r / d \varphi \neq 0$. Thus, for small enough $r_{0}$, the leaves of the foliation $\cos (r)=\tan (r) / \tan \left(r_{0}\right)$ lie entirely under the graph of $\Delta(r, \varphi)=0$, so the discriminant is negative along, and below, the leaves.

Finally, we show that $d r / d \varphi \leq 0$ for $r \geq 0$. This can fail in two ways. We have $d r / d \varphi<0$ near the boundary $\varphi=0$ since $d r / d \varphi=0$ at the boundary and the negative second derivative brings it negative just inside the boundary. If $d r / d \varphi \leq 0$ fails somewhere, then either $d r / d \varphi$ moves up to 0 from below and becomes positive, or $d r / d \varphi$ moves down to $-\infty$ and then becomes positive.

In the first case, if $d r / d \varphi$ approaches 0 from below then at $d r / d \varphi=0$ the second derivative is negative, $d^{2} r / d \varphi^{2}=\lambda^{\prime}(r)<0$, so immediately afterwards, $d r / d \varphi<0$. In the second case, $d r / d \varphi$ cannot approach $-\infty$ since the lower bound $d^{2} r / d \varphi^{2}>\lambda^{\prime}(r)$ prevents $|d r / d \varphi|$ from becoming too big, by the mean value theorem.

Lemma 4.4. A boundary geodesic is convex in a neighbourhood of the boundary.

Proof. The statement of the lemma is equivalent to the following: for small enough $\varphi$ if a geodesic satisfies $d^{2} r / d \varphi^{2}>0$ somewhere then it doesn't meet the boundary at $\varphi=0$. From this we deduce that a boundary geodesic satisfies $d^{2} r / d \varphi^{2} \leq 0$ near the boundary.

As in the proof of Lemma 4.3 we use the cubic polynomial (9). We already pointed out that when $\varphi$ is small enough the cubic polynomial is strictly decreasing since the discriminant (14) is negative.

The unique zero of (9) is negative. If $d^{2} r / d \varphi^{2}>0$ somewhere then $d r / d \varphi$ becomes more negative as $\varphi$ decreases, and $d^{2} r / d \varphi^{2}$ remains positive. In particular, as $\varphi$ decreases so does $d r / d \varphi$. Hence the geodesic cannot meet the boundary with $d r / d \varphi=0$, contradicting Lemma 4.1 . We conclude that $d^{2} r / d \varphi^{2} \leq 0$ in a neighbourhood of the boundary.

Proposition 4.1. There exist geodesics meeting all points of the boundary. 
Proof. Take a sequence of points converging to $(r, \varphi)=\left(r_{0}, 0\right)$ and consider the shortest paths from each of these to the boundary. By Lemma 4.1, when $\varphi$ is small enough the shortest paths are unique. Extend the shortest path to a geodesic of length at least 1 . The remainder of the proof shows that this set of geodesics converges to a geodesic that meets the boundary at $\left(r_{0}, 0\right)$.

The sequence of geodesics $r(\varphi)$ and the sequence of derivatives $d r / d \varphi$ are equicontinuous families so by Arzela-Ascoli converge uniformly to a geodesic. Equicontinuity follows from boundedness and Lemmas 4.3 and 4.4 which supply a uniform bound on the second derivatives $d^{2} r / d \varphi^{2}$ of the sequence of geodesics.

It remains to show that the limiting geodesic meets the boundary at $\left(r_{0}, 0\right)$. We will show that the target of each shortest geodesic lies close to $\left(r_{0}, 0\right)$.

In Lemma 4.4 it was shown that when $\varphi$ is small enough the polynomial (9) has a unique zero. We can estimate it as follows. Let $\delta$ be any small positive number. Express (9) as

$$
\frac{d^{2} r}{d \varphi^{2}}=\frac{1}{\lambda(r)}\left(-\frac{\cos \varphi}{\sin \varphi} \frac{d r}{d \varphi}+\frac{3}{2} \lambda^{\prime}(r)\right)\left(\frac{d r}{d \varphi}\right)^{2}-\frac{\cos \varphi}{\sin \varphi} \frac{d r}{d \varphi}+\lambda^{\prime}(r)
$$

so if $d r / d \varphi=-\delta$ then for small enough $\varphi$ this expression is positive. Since (9) is negative for $d r / d \varphi=0$, the unique zero lies in the interval $(-\delta, 0)$.

From a point, say $\left(r_{0}, \varphi\right)$ for $\varphi$ chosen small enough as in the previous paragraph, the closest point on the boundary satisfies $r>r_{0}$ since $d r / d \varphi<$ 0 . It also lies under any straight line $r / \varphi=c<-\delta$ since the tangent direction to the shortest path must satisfy $d r / d \varphi>-\delta$ so that $d^{2} r / d \varphi^{2}<0$ and by the convexity proven in Lemma 4.4, the geodesic lies under its tangent line.

Thus, as $\varphi \rightarrow 0$, the target points on the boundary converge to $\left(r_{0}, 0\right)$.

Lemma 4.5. The geodesics that meet the boundary meet the line $r=0$ at arbitrarily small angles.

Proof. We have a geodesic running from $\left(0, \varphi_{0}\right)$ to $\left(r_{0}, 0\right)$ satisfying $d r / d \varphi \leq 0$ and $d^{2} r / d \varphi^{2}>\lambda^{\prime}(r)$ by Lemma 4.3. Put $d r / d \varphi=\alpha$ at $r=0$ and recall that $d r / d \varphi=0$ at $r=r_{0}$. By the mean value theorem, there exists $0<r_{1}<r_{0}$ with $d^{2} r / d \varphi^{2}=\alpha / \varphi_{0}$ and since $\varphi_{0}<\pi / 2$ and $\alpha<0$ we get $d^{2} r / d \varphi^{2}<2 \alpha / \pi$ so by $(13)$,

$$
\lambda^{\prime}(r) \pi / 2<\alpha<0
$$


When $r_{0}$ is small, $\lambda^{\prime}(r)$ is close to 0 for $0<r<r_{0}$ so $\alpha$ is arbitrarily small.

Reflect the metric in $r=-\epsilon / 2$ and note that $\lambda(r)=\lambda(-\epsilon-r)$ is still a smooth function. Take the geodesic that meets the boundary at $(r, \varphi)=$ $\left(r_{0}, 0\right)$. By Lemmas 4.2 and 4.5, it meets the line $r=0$ at a value $\varphi_{0}<\pi / 2$ with $d r / d \varphi=\delta<0$. If it also meets the point $(r, \varphi)=\left(-\epsilon, \varphi_{0}\right)$ with $d r / d \varphi>0$ then $d r / d \varphi$ is necessarily $-\delta$ and the geodesic meets the boundary also at $\left(-\epsilon-r_{0}, 0\right)$. The next lemma shows this occurs infinitely often.

Lemma 4.6. There exists a countable set of geodesics with arbitrarily high index that meet the boundary twice.

Proof. Fix $\epsilon>0$. When a geodesic meets $r=0$ at $\varphi=\varphi_{0}$ with $d r / d \varphi=\alpha<$ 0 , then if $\alpha$ is small enough there are finitely many values $r_{i},-\epsilon<r_{i}<0$ such that the geodesic passes through $\left(r_{i}, \varphi_{0}\right)$ with $d r / d \varphi=-\alpha$. Order these points with $0>r_{1}>r_{2}>\ldots>r_{k} \geq-\epsilon$. The difference $r_{i}-r_{i+1}$ gives the period and is constant. The quantity $\left(r_{k}+\epsilon\right) /\left(r_{i}-r_{i+1}\right) \in[0,1)$ varies continuously with the point $r_{0}$, where the geodesic meets the boundary. For $r_{0}$ small enough, the angle between the geodesic and $r=0$ is small and so is the period. Thus we can choose $r_{0}$ small enough that there are more than $k$ values $r_{i}$ defined above. By the intermediate value theorem, there is a value of $r_{0}$ in between such that the quantity $\left(r_{k}+\epsilon\right) /\left(r_{i}-r_{i+1}\right)=0$. This geodesic meets the boundary also at $\left(-\epsilon-r_{0}, 0\right)$. This construction produces such a geodesic for arbitrarily small $r_{0}$ and hence arbitrarily high index.

Lemma 4.7. If $\lambda$ satisfies (12) and $\lambda^{\prime \prime}(r) \leq 2 \sin ^{2} r$ for $r>0$ then the Ricci curvature of the metric on the three-sphere is non-negative.

Proof. The Ricci curvature of the metric is

$$
\left(\begin{array}{ccc}
\frac{1}{2} \lambda^{\prime}(r)^{2} / \lambda(r)^{2}-\lambda^{\prime \prime}(r) / \lambda(r) & 0 & 0 \\
0 & \left(1-\frac{1}{2} \lambda^{\prime \prime}(r)\right) / \lambda(r) & 0 \\
0 & 0 & \left(1-\frac{1}{2} \lambda^{\prime \prime}(r)\right) / \lambda(r)
\end{array}\right) .
$$

In a neighbourhood of $\lambda(r) \equiv 1$ the first term of the Ricci curvature vanishes and the other two terms are 1. For $r>0$ (and $r<-\epsilon$ ), if $\lambda^{\prime \prime}(r)<0$ then clearly each term is positive. Thus, assume $0 \leq \lambda^{\prime \prime}(r) \leq 2 \sin ^{2} r$. Together with $\lambda(r)<\cos ^{2} r$ we get $-\lambda^{\prime \prime}(r) \lambda(r)>-2 \sin ^{2} r \cos ^{2} r$. Since 
$\lambda^{\prime}(r)^{2}>\sin ^{2} 2 r$, then $(1 / 2) \lambda^{\prime}(r)^{2}-\lambda^{\prime \prime}(r) \lambda(r)>0$ and the first entry of the Ricci curvature is positive. The other two entries are bounded below by $\cos ^{2} r / \lambda(r)>0$.

Since the geodesics that meet the boundary arbitrarily close to $r=0$ are the important ones, we can take a part of the surface $r>r_{0}$ that is untouched by the family of geodesics. This corresponds to a ball in the three-sphere. We can cut out this ball and glue in a three-manifold so the minimal surfaces live in any three-manifold.

In the proof of the previous lemma it is shown that the Ricci curvature is positive when $\lambda^{\prime \prime}(r)>0$. Thus, in the interval $-\epsilon \leq r \leq 0$ we can increase the sizes of the two-spheres to get positive Ricci curvature. That is, there is an arbitrarily small deformation of $\lambda(r)$ in a neighbourhood of $-\epsilon \leq r \leq 0$ with $\lambda^{\prime \prime}(r)>0$. Equivalently, we can embed the three-sphere as a boundary of a convex ball in $\mathbb{R}^{4}$ :

$$
x_{1}^{2}+x_{2}^{2}+x_{3}^{2}=F\left(x_{4}\right)
$$

where $F\left(x_{4}\right)=\lambda(r)$ and $x_{4}$ and $r$ are related by

$$
\left(\frac{d x_{4}}{d r}\right)^{2}=1-\frac{(d \lambda / d r)^{2}}{4 \lambda}
$$

and $x_{4}(r=0)=0$. The deformation above deforms the ball to be strictly convex.

As mentioned in the introduction, positive Ricci curvature bounds the area, and hence index, of each embedded minimal sphere.

\section{References.}

[1] Choi, H. and Schoen, R. The space of minimal embeddings of a surface into a three-dimensional manifold of positive Ricci curvature. Inv. Math. 81 (1985), 387-394.

[2] Colding, T. and Hingston, N. Metrics without Morse index bounds, To Appear in Duke Math. Journal.

[3] Colding, T. and Minicozzi, W. Examples of embedded minimal tori without area bounds, Internat. Math. Res. Notices 20 (1999), 1097-1100.

[4] Colding, T. and Minicozzi, W. Minimal surfaces, Courant Lecture Notes in Mathematics, NYU, Courant Institute of Mathematical Sciences, (1999). 
[5] Hsiang, W. and Lawson, H.B. Minimal submanifolds of low cohomogeneity, J. Diff. Geom. 5 (1971), 1-38.

[6] Milnor, J. Morse Theory, Annals of Mathematics Studies 51. Princeton, N.J., Princeton University Press (1963).

[7] Pitts, J.T. and Rubinstein, J.H. Applications of minimax to minimal surfaces and the topology of 3-manifolds, Miniconference on geometry and partial differential equations, 2 (Canberra, 1986), Proc. Centre Math. Anal. ANU, 12 (1987), 137-170.

[8] Simons, J. Minimal varieties in Riemannian manifolds, Ann. Math. (2) 88 (1968), 62-105.

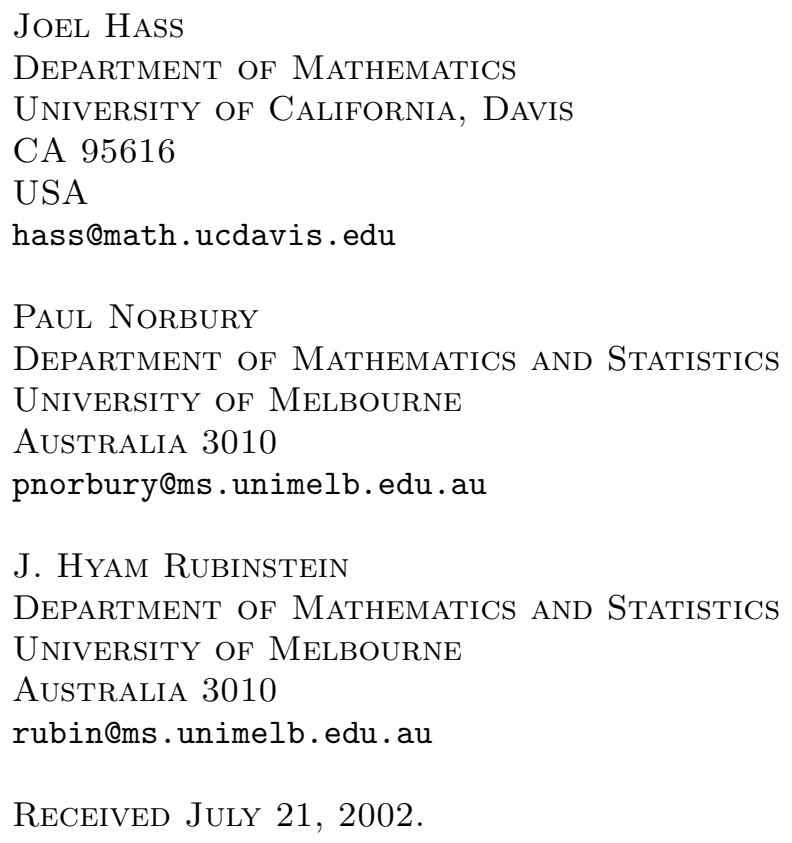

